Offizielles Organ von

DGHO - Deutsche Gesellschaft für Hämatologie und Onkologie

ÖGHO - Österreichische Gesellschaft für Hämatologie und Onkologie

$\mathrm{DFaG}$ - Deutsche Fatigue Gesellschaft

AIO - Arbeitsgemeinschaft Internistische Onkologie in der deutschen Krebsgesellschaft e.V.

Mitglied der Deutschen Krebsgesellschaft e.V.

\title{
Editors
}

Editor-in-Chief

H.-J. Schmoll, Halle
Clinical Cases

R. Hartenstein, München

Dermatologic Oncology

A. Hauschild, Kiel

Drug Development

T. Cerny, St. Gallen

Gynecologic Oncology

R. Kreienberg, Ulm

Genitourinary Oncology

H. Rübben, Essen
Hematological Malignancies

M. Hallek, Köln

Immunotherapy

G. Gastl, Innsbruck

Medical Oncology

C. Bokemeyer, Hamburg

Radiation Oncology

T. Wendt, Jena

Pediatric Oncology

H. Jürgens, Münster

Surgical Oncology

P.M. Schlag, Berlin

K. Jordan, Halle

Hematology and Medical Oncology

L. Bergmann, Frankfurt/M.

M. Borner, Bern

A. Engert, Köln

S. Fruehauf, Osnabrück

E. Gunsilius, Innsbruck

T. Haferlach, München

D. Haller, Philadelphia

J.T. Hartmann, Tübingen

U. Jäger, Wien

U.R. Kleeberg, Hamburg

G. Kornek, Wien

H.-E. Laack, Hamburg

A. Mackensen, Erlangen

R. Morant, St. Gallen

K. Mross, Freiburg i.Br.

K. Possinger, Berlin

W. Scheithauer, Wien

L. Trümper, Göttingen

J.B. Vermorken, Edegem

Oncological Rehabilitation

H.H. Bartsch, Freiburg i.Br.

Palliative Care

C. Bausewein, London

I. Strohscheer, Hamburg

Pathology

H.E. Gabbert, Düsseldorf
Pediatric Oncology

J. Boos, Münster

H. Gadner, Wien

U. Göbel, Düsseldorf

Pharmacogenomics, -kinetics, -dynamics

M. Czejka, Wien

H.-J. Lenz, Los Angeles

Psycho-Oncology

F.A. Muthny, Münster

M. Neises, Aachen

Radiation Oncology

W. Budach, Düsseldorf

G. Hartung, Heidelberg

P. Lukas, Innsbruck

R.O. Mirimanoff, Lausanne

A.U. Schratter-Sehn, Wien

F. Wenz, Mannheim

T. Wiegel, Ulm

Surgical Oncology

D. Henne-Bruns, Ulm

K.W. Jauch, München

P. Macchiarini, Barcelona

B. Nordlinger, Boulogne

H.-D. Saeger, Dresden

N. Senninger, Münster

Tumors of the Head and Neck

F. Bootz, Bonn

H.-P. Howaldt, Gießen

J.A. Werner, Marburg

H. Kölbl, Mainz

I.B. Runnebaum, Jena

\section{Editorial Office}

S. Karger GmbH

Attn. Dr. Steffi Hentzelt

P.O. Box

D-79095 Freiburg

E-mail s.hentzelt@karger.de 